\title{
NEUE PROBLEME UM DEN TODESBEGRIFF IM STRAFRECHT DER BUNDESREPUBLIK DEUTSCHLAND *
}

\author{
von Professor Dr. hur. Hans Lüttger, \\ Freie Universität Berlin.
}

Die drei Zäsuren, die den strafrechtlichen Lebensschutz abgrenzen, haben in der deutschen Rechtswissenschaft lange Zeit hindurch das Bild einer beschaulichen Ruhe geboten. In wenigen Jahren hat sich dies drastisch geändert : Bei der Frage nach dem Beginn des strafrechtlichen Lebensschutzes hat die biologisohe Station der Nidation in der Rechtslehre zunehmend diejenige der Befruchtung verdrängt, die ein Jahrhundert lang unangefochten den Beginn des Leibesfruchtstadiums und damit die Anfangszäsur der Strafbarkeit wegen Abtreibung bezeichnet hat. An der Grenze zwischen Leibesfruchtcharakter und Menschqualität hat der Contergan- Prozess eine Fülle von Rechtsfragen auf geworfen, die generationenlang unproblematisch erschienen sind, nun aber dazu zwingen, die Abschichtung des Strafschutzes vor und nach der Zäsur "Beginn der Geburt" dogmatisch klarer herauszuarbeiten. Und auch das Ende des strafrechtlichen Lebensschutzes, der Tod, ist zu einem juristischen Problem par excellence geworden. Die Zeit, in der auch renommierte Lehrbuicher des Strafrechts sich auf den lapidaren Satz beschränken konnten "Die Menschqualität endet mit dem Tode», ist vorbei. Denn des, was wir rechtlich unter «Tod» zu verstehen haben, ist nicht mehr selbstverständlich. Darum geht es im folgenden. Auch hier wird sich -ebenso wie bej den anderen Zäsuren im strafrechtlichen Lebensschutz- die enge

\footnotetext{
* Der Vortrag gibt in überarbeiteter Fassung ein Kapitel aus einem Referat wieder, das der Verfasser am 19. März 1970 auf einer Tagung der Deutschen Richterakademie im Berlin gehalten hat. Der Vortrag ist bereits in Juristische Rundschau (Berlin) 1971, S. 309 ff veröffentlicht. Dort finden sich umfassende Hinweise auf die Rechtsprechung sowie auf die juristische und medizinische Literatur.
} 
Verflechtung zwischen medizinischen und rechtlichen Überlegungen zeigen.

A.

Das deutsche Recht kennt -wie anscheinend die meisten ausländischen Rechtssysteme- keine Legaldefinition des Todesbegriffs und legt auch keine Kriterien fest, nach denen der Zeitpunkt des Todeseintritts zu bestimmen wäre. Das gilt nicht nur für die grossen Kodifikationen, in denen -wie im Strafrecht und im Bürgerlichen Recht- vorn «Tode» die Rede ist; es gilt vielmehr auch für jene Spezialmaterien, die sich mit den amtlichen Folgen eines Todesfalles befassen. So wollen die Vorschriften des Bestattungsund Leichenschaurechts nur gewährleisten, dass eine Beerdigung oder Einäscherung erst nach sicherer Feststellung des Todes und gegebenenfalls der Todesursache erfolgt; und die Vorschriften des Personenstandsrechts wollen nur die Anzeige und die standesamtliche Eintragung von Sterbefällen sichern. Aber beide definieren nicht den Todesbegriff und dekretieren nicht, was als Todeszeitpunkt zu gelten habe. Der Rechtsbegriff des «Todes» ist also ein offener Begriff, der nach den Erkenntnissen der medizinischen Wissenschaft und den Regeln juristischer Interpretation auszufüllen ist.

1. Bis vor wenigen Jahren hat die deutsche Strafrechtswissenschaft - - scweit sie sich überhaupt mit unserer Frage befasst hat-- unter dem «Tod» des Menschen das endguiltige Aufhören von Herztätigkeit und Atmung verstanden. Dieser - sehr lapidarenFormel lag unverkennbar der sogenannte "klassische Todesbegriff" zugrunde, der in der Medizin bis vor wenigen Jahren unangefochten galt. Die medizinische Wissenschaft umschrieb ihn freilich in naturwissenschaftlicher Hinsicht viel genauer; sie definierte den Tod nämlich als den «irreversiblen (unumkehrbaren) Stillstand von Kreislauf und Atmung, verbunden mit dem Aufhören der Tätigkeit des Zentralnervensystems und gefolgt vom Absterben aller Zellen und Gewebe des Organismus»'. An dieser klassischen Todesdefinition fällt. ein Doppeltes auf : Einerseits trug sie der biologischen Einsicht Rechnung, dass der Tod kein «abruptes Ereignis», sondern ein "fortschreitender Prozess» ist und dass zwischen Leben und Tod der allmähliche «Ưbergang des Sterbens» liegt, währenddessen die Lebensfunktionen nacheinander erlöschen und Zelle auf Zelle, Organ auf Organ abstirbt. Anderseits klang aber in dieser

I Vgl. Hansen, Gerichtliche Medizin, 2. Auflage 1965, S. 20 und 22. 
klassischen Todesdefinition auch an, dass der irreparable Ausfall von Kreislauf und Atmung praktisch das entscheidende Einzelereignis in diesem Vorgang des Sterbens war; denn nunmehr schloss sich ja der endgültige Auflösungsprozess unentrinnbar an, weil die Zellen des menschlichen Körpers nach dem Ausfall der Sauerstoffversorgung nur eine sehr begrenzte thberlebensfähigkeit haben. Wenn also die medizinische Praxis und die Rechtslehre versuchten, in dem Prozess des Stenbens denjenigen Zeitpunkt zu fixieren, der den Tod symbosisiente, so war dies unverkennbar der irreversible Ausfall von Kreislauf und Atmung, nach dem es kein «Zurück ins Leben» mehr gab. Damit war eine praktikable rechtliche Endzäsur des strafrechtlichen Lebensschutzes gefunden, die dem damaligen Stande der ärztlichen Möglichkeiten entsprach'2

2. Mit der Entwicklung der modernen Reanimationstechniken und mit der apparativen Ersetzbarkeit von Herztätigkeit und Atmung komplizierte sich indessen die Lage.

Freilich waren manche aus den modernen medizinischen Techniken abgeleiteten Bedenken gegen die klassische Todesdefinition allzu voreilig und vordergründig. So wissen wir seit langem, dass eine -etwa bei Unfällen oder bei Operationszwischenfällenaussetzende Herz- und Atmungstätigkeit durch schleunige Massnahmen oftmals wieder in Gang gesetzt werden kann, von denen die Mund-zu-Mund-Beatmung und die Herzmassage allseits bekannt sind. Haben diese Massnahmen Erfolg, so könnte nur derjenige von einem "zum Leben erweckten Toten" sprechen, der das Merkmal der Irreversibilität in der klassischen Todesdefinition fälschlich ausser acht lassen würde. Nichts anderes gilt aber für die erfolgreiche Anwendung sonstiger neuartiger Reanimationstechniken. Der klassische Todesbegriff versagte hier keineswegs; nur die ¿Umkehrbarkeit" wurde um weitere Amwendungsfälle bereichent.

Und auch der Ersatz des spontanen Herzschlags durch sogenante Schrittmacher und der Ersatz der Spontanatmung durch Respiratoren brauchte zunächst nur zu einer Verdeutlichung dahingehend zu führen, dass es für die klassische Todesdefinition nicht auf Spontaneität (Selbständigkeit) von Kreislauf und Atrnung ankommot ${ }^{2}$. Denn welcher vernünftige Mensch wollte von einem "Toten» reden, wenn ein infolge Poliomyelitis Gelähmter künstlich beatmet wird oder wenn während einer Herzoperation der eigene

2 VgI. zum Ganzen: Geilen in Zeitschrift für das gesamte Familienrecht (FamRZ) 1968, S. 123 ff, tund in Juristenzeitung (JZ) 1968, S. 149 ff. 
Kreislauf und die eigene Atmung des Patienten ausgeschaltet und apparativ ersetzt werden? Die rechtliche Folge dieser technischen Entwicklung wäre also zunächst nur, dass jeder Stillstand von Kreislauf und Atmung, der künstlich wieder aufgehoben werden könnte, vorläufig als "reversibler» Stillstand bezeichnet werden müsste, unabhängig davon, ob die Funktion dann selbständing in Gang bleiben könnte ${ }^{3}$.

Die entscheidenden Bedenken gegen die klassische Todesdefinition liegen daher auch auf einem anderen Gebiet. Die Zellen des menschlichen Körpers haben -wie insbesondere die Erfahrung mit den Reanimationstechniken gezeigt hat- eine sehr unterschiedliche "Wiederbelebungszeit»; genauer gesagt: Die Zeitspanne, welche sie ohne Zufuhr von frischem, sauerstoffhaltigem Blut überleben können, divergiert beträchtlich4. So stirbt beim Ausfall von Kreislauf und Atmung das Gehirn bereits nach 3 bis 4 Minuten ab; seine Uberlebensfrist kann sich nur unter besonderen Bedingungen -beispielsweise bei Kindern, bei starker Untenkühlung und unter Hypnotika oder Narkatika - maximal auf 8 his 12 Minuten verlängern. Demgegenüber haben andere Organe -wie Leber, Lunge, Galle, Herz und Nieren- längere Wiederbelebungsfristen. Für das Herz beispielsweise wird die Überlebensfrist von medizinischer Seite zwischen 30 Minuten und 1 1/2 Stunden angegeben, wobei es freilich ebenfalls entscheidend auf die Bedingungen ankommt und auch mit fortschreitender Dauer Funktionsschwächen zurückbleiben. Jedenfalls ergibt sich aus dieser Diskrepanz der Uberlebensfristen, dass eine verspätet einsetzende Reanimation den Kreislauf und die Atmung -wenigstents künstlich- wieder in Gang setzen kann, während das Gehirn schon unwiderruflich zerstört ist. -Ein solcher Befund kann sich aber nicht nur bei einem derartigen "natürlichen" Todesverlauf einstellen, sondern auch in dem- gewissermassen umgekehrten -Falle, wenn das Gehirn infolge äusserer oder innerer Ursachen unmittelbar getroffen wird, ohne dass zuvor Kreislauf und Atmung sistiert waren. Das ereignet sich namentlich bei Gehirnzertrümmerungen durch äussere Gewalteinwirkung und bei intrakraniellen Blutungen $(=$ Blutungen innerhalb des Schädels), die zu akuter Drucksteigerung und zur Zerstörung der Hirnsubstanz führen. In diesen Fällen, die für eine

${ }^{3}$ Vgl. Spann, Deutsche Zeitschirft für die gesamte gerichtliche Medizin (DZGerMed) 1966, S. 28.

4 Vgl. zum folgenden: Stratenwerth in Festschrift für Engisch, 1969, S. 528

ff; Heinitz, Rechtliche Fragen der Organtransplantation, 1970, S. 13 ff. 
Organtransplantation besondere Bedeutung haben, lassen sich dann oftmals durch schleunigen Einsatz von Reanimationsmethoden zwar die übrigen Organe (wie etwa das Herz), nicht aber das Gehirn "retten». --Beiden Fallgruppen ist indessen gemeinsam, dass Kreislauf und Atmung zwar iiber eine gewisse Zeit hinweg aufrechterhalten werden können, dass jedoch der endguiltige Zerfall nach einem kürzeren oder längeren Intervall unaufhaltsam eintritt ${ }^{5}$.

Wollte man in diesen Fällen mit der klassischen Todesdefinition auf den irreversiblen Ausfall der (nicht notwendig spontanen) Herztätigkeit und Atmung abstellen, so wäre der Körper mit totem Gehirn, aber apparativ aufnechterhaltenem Kreislauf und Atmung ein «lebender Mensch». Das hätte eine unausweichliche Konsequenz: Die Entnahme von lebenswichtigen unpaarigen Organen aus einem Körper mit abgestorbenem Gehirn, aber nich aufrechterhaltenem Kreislauf und Atmung wäre rechtlich Tötung eines "Lebenden». Bieharrt man nämlich auf dem klassischen Begriff des Herz- und Atmungstodes, so gilt bis zu dessen Eintritt nach deutschem Recht unverbruichlich: Der strafrechtliche Lebensschutz kommt auch dem unheilbar Kranken, dem Todgeweihten und dem Sterbenden zugute. Und es gibt im deutschen Recht keinen Rechtfertigungsgrund, der es dem Arzt gestatten könnte, durch den aktiven Eingriff einer todbringenden Transplantatentnahme das Leben eines moribundus zugunsten eines anderen $\mathrm{Pa}$ tienten zu opfern ${ }^{6}$. Das Ende der Transplantation von lebenswichtigen unpaarigen Organen wäre dann gewi $\beta$; denn dafür sind nur "lebensfrische» funktionstüchtige Organe verwendbar.

3. Der geschilderte medizinisch-technische Fortschritt und die dabei gewonnenen Erkenntnisse haben geradezu zwangsläufig die Frage aufgeworfen, ob der klassische Begriff des «Herz- und Atmungstodes» durch einen neuen Todesbegriff -nämlich den des «Gehirntodes»- ablösbar sei. Das Ergebnis ist bekannt : Nicht nur die medizinische Wissenschaft, sondern auch die deutsche Strafrechtslehre erkennen heute —-soweit ich sehe : nahezu einmütigden Hirtnod als den maßgeblichen Todeszeitpunkt an. Uns inte-

5 Vgl. dazu insbesondere : Entschließung der Kommission für Reanimation und Organtransplantation der Deutschen Gesellschaft für Chirurgie, in Der Chirurg 1968, S. 196 ff; Richtlinien der Schweizerischen Akademie der Medizinischen Wissenschaften für die Definition und die Diagnose des Todes, in Schweizerische Juristenzeitung 1969, S. 248.

${ }^{6}$ Vgl. dazu : Stratenwerth (wie Anmerkung 4), S. 539 u. 541. 
ressiert jedoch zunächst die Begründung für diese wahrhaft umwälzende Änderung der Rechtsauffassung.

Dabei sei vorweg ganz klar herausgestellt : Der Umstand, da $\beta$ nur eine Verlegung des Todeszeitpunkts auf das Absterben des Gehirns den Weg zur Transplantation von Herzen und manchen anderen Organen eröffnet, könnte allein niemals einen hinreichenden Grund für die Anerkennung des Gehirntodes abgeben. Der strafrechtliche Lebensschutz ist nicht nach solchen "Nützlichkeitserwägungen» manipulierbar. Fände sich keine andere Begründung, so hätten wir das skizzierte Ergebnis hinzunehmen.

a) Für die Anerkennung des Gehirntodes wird sehr oft eine anthropologische Begründung gegeben; lassen wir Zitate dafür sprechen:? Der Mensch ist eine ontologische Einheit aus Geistseele und Leib; er ist auch im rechtlichen Sinne kein Aggregat von Funktionen, sondern ein Ganzes, eine sinnvolle Einheit, eine Person. Der menschliche Geist, der die Einzigartigkeit der menschlichen Individualität bedingt, ist aber das Produkt des Gehirns, nicht des Herzens. Das Gehirn als Sitz unseres Bewußtseins begründet unser individuelles Menschsein; es gewährleistet die geistigen Funktionen und verkörpert das eigentlich Menschliche; es ist die oberste integrierende, bewußtseinstragende und persönlichkeitsbestimmende Instanz. Der Zustand nach dem Tode des Gehirns ist daher unvereinbar mit den Begriffen Leben und Mensch. Entscheidend für das Leben des Menschen kann deshalb nur das Leben seines Zentralorgans Gehirn sein. Mit dem Organtod des Gehirns erlischt die individuelle menschliche Existenz. Der Hirntod bewirkt das Ende der Existenz des Menschen als Person; nur die Person aber kann das Recht als lebendigen Mensohen gelten lassen. Der Organtod des Gehirns ist daher gleichbedeutend mit dem Tode des Menschen; der Zeitpunkt, in welchem die Hirnfunktion erlischt, ist der Zeitpunkt des Todes des Menschen. Der Körper mit irreversibel zer. störtem Gehirn ist kein Mensch mehr; er ist nur noch ein Leichnam mit mehr oder weniger künstlich oder spontan erhaltenen Teilfunktionen, ein überlebendes Präparat. Fontbestehende periphere Organfunktionen sind nur noch animalische Restbestände.

Wenn sich hier trotz eines solchen Aufgebots wohltönender Worte Bedenken regen, dann liegt dies nicht nur an einer "form-

\footnotetext{
${ }^{7}$ Die Fundstellen zu den folgenden Zitaten finden sich bei : Lüttger in Ju-
} ristische Rundschau (JR), 1971, S. 309 ff. 
men Scheu" und nicht nur an dem Mißtrauen gegen pathetische Floskeln, das ich mit vielen Juristen teile. Es müßte nicht einmal an den sich hier aufdrängenden Assoziationen zu Begriffen wie "Lebenswert" und "Lebenssinn" liegen, unter deren Flagge einst ein verbrecherischer Massenmond an sogenanntem «lebensunwertem Leben" getrieben worden ist. Das Unbefriedigende dieser Theorie liegt vielmehr an ihrem enklärten Ansatzpunkt : dem Versuch, zwischen «biologischem" und "menschlichem" Leben nach dem Maßstab der "anthropologischen Relevanz» zu unterscheiden. ${ }^{8}$ Es steht hier nicht zur Debatte, welchen Platz dieser Begriff in außerjuristischen Disziplinen hat; hier geht es nur darum, ob dieser Begriff inhaltlich wenigstens so viel Konturen besitzt, da $\beta$ er als rechtlicher Maßstab tragbar wäre. Versteht man ihn im her. kömmlichen Sinne als die -mindestens potentielle-_ Fähigkeit zur Kommunikation oder als -mindestens potentielles- Wirksamwerden der Geistseele, so ist gerade nicht verbürgt, daß dem Leben éines in irreparabler Bewußtlosigkeit «dahinvegetierenden» Menschen eben jene anthropologische Relevanz zugemessen wird. Es ist sicher kein Zufall, daß einezlne namhafte Juristen und Mediziner bereits fälschlich schwere Hirnschädigungen und irreversiblen Bewußtseinsverlust als "Gehirntod" deklariert haben. Der Begriff der anthropologischen Relevanz ist offenbar zu unbestimmt, als da $\beta$, er die Warnung vor der Gefahr, im übertragenen weiteren Sinne «geistig Toten» das Lebensrecht abzusprechen, vergessen machen könnte. Aus der zuttreffenden Interpretation von heute kann der Mißbrauch von morgen werden.

Freilich wollen -abgesehen von den erwähnten Einzelstimmen- die Vertreter dieser Theorie die anthropologische Relevanz nur verneinen, wenn das Gehirn total und irreversibel zerstört ist. Dabei wird dann aber offenbar, $\mathrm{da} \beta$ es auf solche Überlegungen gar nicht ankommt.

b) Die Anerkennung des Hirntodes als des für den Tod des Menschen entscheidenden Ereignisses ist nämlich aus rein medizinisch-juristischen Gründen gerechtfertigt.

Schon die alte juristische Definition des Herz- und Atmungstodes griff aus dem Proze $\beta$ des Sterbens denjenigen Zeitpunkt und das Einzelereignis heraus, welche den Tod des Menschen symbo.

8 Vgl. dazu : Geilen, FamRZ 1968, 127, und JZ 1968, 151; Kautzky in Hoch. land $1960 / 61$, S. 314. 
lisierten; nach dem Stande der damaligen ärztlichen Möglichkeiten war dies der irreversible Ausfall von Kreislauf und Atmung. Indessen ist der Ausfall von Herztätigkeit und Atmung weitgehend reversibel geworden; das Herz ist heute -wie die Transplantationen zeigen - auswechselbar; und bei vielen Kranken muß die Atmung über lange Zeit hinweg apparativ ersetzt werden. Haute wäre es geradezu willkürlich, das Aufhören von Atmung und Herzschlag als Todeskriterium anzusehen. Ganz anders liegt es beim Ausfall des Gehirns: Das Erlöschen der Gehirnfunktionen ist irreversibel; das Gehirn ist apparativ nicht ersetzbar; nach dem Tode des Gehirns vermag auch die Reanimation den endguiltigen Zerfall nur aufzuschieben, nicht aber zu verhindern. Mit diesen Einsichten ist die Rolle, die im Proze $\beta$ des Sterbens bislang der Ausfall von Kreislauf und Atmung gespielt hat, dem Erlöschen des Gehirns zugefallen : es symbolisiert den Tod des Menschen. Daran rechtlich anzuknüpfen, ist legitim. Denn einmal braucht das Recht —ebenso wie die praktische Medizin- einen Todesbegriff, der sich als Zäsur und nicht als Proze $\beta$ versteht. ${ }^{9}$ Zum anderen ändert sich an der Methodik der Todesbestimmung gar nichts : es bleibt bei der Lokalisierung und der zeitlichen Fixierung auf ein irreversibles und daher entscheidendes Einzelereignis - nur ist dies jetzt der endguiltige und totale Ausfall des Gehirns. Hält man aber an der -unbestritten geibten- juristischen Methodik solcher Todes-bestimmung fest, dann muß das Recht nunmehr den Hirntod anerkennen. Denn mit dem Rechtsbegriff des Todes kann nur ein absolut unumkehrbarer Endpunkt des Lebens gemeint $\operatorname{sein}^{10}$.

Infolgedessen ist es in juristischer Sicht auch unrichtig, wenn nicht selten empfohlen wird, sich im "Normalfall» weiterhin mit der klassischen Definition des Herz- und Atmungstodes zu begnüigen und nur in Reanimationsfällen auf den Gehirntod abzustellen. Praktisch wünde dies zwar meist keine nennenswerten Auswirkungen haben; denn der Gehirntod folgt dem Stillstand von Kreislauf und Atmung ohne schleunige Reanimation notwendig binnen weriger Minuten nach. Aber dies kann doch nicht dariber hinwegtäuschen, daß der Ausfall von Kreislauf und Atmung seine juristischdefinitorische Bedeutung verloren hat und $\mathrm{da} \beta$ statt desses der Gehirntod jenes absolut irreversible und daher entscheidende

\footnotetext{
${ }^{9}$ Vgl. dazu Geilen wie Anm. 2.

${ }^{10}$ So mit Recht: Richard Lange in Leipziger Kommentar zum Strafgesetzbuch (LK), 9. Auflage 1970/71, Randziffer (Rdz) 4 der Vorbemerkung vor § $211 \mathrm{StGB}$.
} 
Ereignis ist, welches nunmehr im Rechtssinne allein den Tod des Menschen darstellt. Ganz abgesehen von der juristischen Absurdität, zwei verschiedene Todesbegriffe nebeneinander zu praktizie. ren.

4. Fassen wir also unser bisheriges Ergebnis zusammen : Das Recht kann diesen neuartigen Begriff des «cerebralen Todes» iiber. nehmen, weil der Rechtsbegriff des Todes ein «offener Begriff» ist. Nicht unsichere anthropologische Wertungen, sondern medizinische Fakten füllen diesen Begriff aus und rechtfertigen den Wandel in der Rechtsauffassung. Begreift man den Tod als rechtliche Zäsur, so bleibt gar keine andere Wahl, als auf das irreversible und entscheidende Ereignis des absoluten und endgiiltigen Erlöschens der Hirnfunktionen abzustellen: Der Hirntod mu $\beta$ nunmehr an die Stelle des Herz-- und Atmungstodes treten.

Doch ist es angezeigt, den Begriff des "Gehirntodes" noch deutlicher zu beschreiben: Unter dem Organtod des Gehirns ist "die grobanatomische oder feinstrükturelle Zerstörung des Gehirns in seiner Gesamtheit» zu verstehen". Daraus folgt ein Mehrfaches : Nur eine Zerstörung des Gehirns in seiner Gesamtheit (Hirnrinde und Stammhirn) füllt den neuen Todesbegriff aus, niemals eine -wenn auch schwere- Hirnschädigung, wie sie sich beispielsweise in irreparabeler Bewusstlosigkeit äußern kann. Und weiter mu $\beta$ dieser Zustand der totalen Gehirnzerstörung definitiv erreicht sein; denn «ein sterbendes Hirn ist kein totes Gehirn wie ein sterbender Mensch kein Leichnam" ist $t^{12}$. Ist aber das Gehirn in seiner Gesamtheit definitiv zerstönt, so ist der Tod des Menschen da, selbst "wenn in der leiblichen Hülle das Herz noch schlägt»" Wenn jetzt einzelne Organe - mit oder ohne Reanimation- den Hirntod für kürzere oder längere Zeit überleben, so sind sie in der Tat nichts anderes als «Iebende Organpräparate». Das gab es schon immer; denn auch nach dem Ausfall von Herztätigkeit und Atmung überleben ja die einzelnen Organe noch Minuten und sogar Stunden, ohne daß deshalb der - ebenfalls als lokalisierte und zeitlich fixierte Züsur verstandene- Rechtsbegriff des Herz- und Atmungstodes rechtlich jemals angezweifelt worden wäre. Wir haben

11 Vgl. dazu die in Anm. 5 zitierte Entschließung der Deutschen Gesellschaft für Chirurgie.

12 Vgl. Blei in Juristische Arbeitsblätter 1970, S. 270.

${ }^{13}$ Vgl. dazu und zum folgenden: Bockelmann, Strafrecht des Arztes, 1968, S. 109 . 
uns im Gegenteil heute noch weit mehr mit der Vorstellung von überlebenden Organen, Geweben und anderen Körperbestandteilen vertraut gemacht, seien es nun Blutkonserven, Präparate in Organbanken oder aus einem Toten auf einen Lebenden transplantierte "weiterlebende" Organe. Ein als Zäsur verstandener Rechtsbegriff des Todes erlaubt es uns mithin, zwischen «lebendem $\mathrm{Ge}$ webe» und dem «Leben des Menschen» zu unterscheiden.

B.

Damit sind jedoch die Probleme, die durch die Anerkennung des Gehirntodes aufgeworfen worden sind, nicht zu Ende; sie beginnen im Grunde erst bei der Frage nach der Feststellung des Gehirntodes. Denn es ist selbstverständlich, da $\beta$ der Eintritt des Gehirntodes schon aus Rechtsgründen des sicheren Beweises bedarf, sobald eine Entnahme von lebenswichtigen Transplantaten in Betracht kommt. Hier bestehen nach wie vor große Schwierigkeiten.

Dabei sei vorweg unmißverständlich klargestellt : Ganz indiskutabel ist der vereinzelt gemachte Vorschlag, in denjenigen Fällen, in welchen der Kreislauf zum Zwecke der Organtransplantation künstlich aufrecht erhalten wird, den Tod zu dem Zeitpunkt als eingetreten anzusehen, an welchem der Arzt oder ein Ärztegremium der Überzeugung sei, da $\beta$ ein selbständiges, von Wiederbelebunsgeräten unabhängiges Leben nicht mehr wiederhergestellt werden könne. Ganz abgesehen davon, daß hier eine Anknüpfung an den Gehirntod nicht mehr erkennbar wird, ist diese Subjektivierung des Todesbegriffs mit Recht auf Ablehnung gestoßen : Das Recht braucht objektive Todeskriterten, wenn nicht der Todesbegriff relativiert und seine Feststellung der breiten Skala vom irrenden Gewissen bis zum gewissenlosen Mißbrauch anheimfallen soll.

1. Um solche objektive Kriterien für die Feststellung des Gehirntodes bemiiht sich die medizinische Wissenschaft seit Jahren mit verantwortungsvollem Ernst. Das Dilemma besteht darin, da $\beta$ es keine verbindlichen Einzelsymptome gibt, welche klinisch die Diagnose des Gehirntodes gestatten, und da $\beta$ auch die diagnostischen Hilfs-Methoden nicht frei von Fehlerquellen sind. So erwächst denn die ärztliche Feststellung über den Hirntod aus einem klinisch-neurologischen Gesamturteil, das durch bestimmte diagnostische Hilfen gestützt wird und mannigfache Symptome berücksichtigen muß. Ich kann diese medizinischen Aspekte unseres Be. weisproblems hier nur kurz andeuten. 
a) Die bekanniteste Hilfsmethode bei der Festellung des Gehirntodes ist das sogenannte Electro-Encephalogramm (EEG) ${ }^{14}$. Mit ihm können die bioelektrischen Vorgänge, die sich im lebenden Gehirn abspielen, normalerweise als Hirnstromkurve sichtbar gemacht werden. Im Falle des Gehirntodes zeigt das EEG -infolge der bioelektrischen Stille im Gehirn- eine "Null-Linie» an. Indessen kann aber umgekehrt nicht allein aus der Null-Linie im EEG auf Gehirntod geschlossen werden, und zwar aus mehreren Griinden : Zunächst gibt das EEG nur Aufschluß über die Funktion von Teilen der Hirnrinde; es gestattet also keinen șicheren Schluß auf den Zustand der tiefer gelegenen Hirnteile, insbesondere des Stammhirns. Zum Begriff des Gehirntodes gehört aber -wie erörtertdie Zerstörung des Gehirns in seiner Gesamtheit; schon deshalb bedarf es also neben dem EEG ergänzender neurologischer Untersuchungen über den Ausfall des Stammhirns. - Sodanın kann die Null- Linie im EEG auch das Zeichen einer reversiblen Funktionsblockierung des Gehirns sein; die Stille im EEG kann also buchstäblich einen "Scheintod" anzeigen. -Und schließlich besteht auch keine Einigkeit dariber, wie lange und wie oft hirnelektrische Stille im EEG gemessen sein mu $\beta$, um überhaupt einen (begrenzten) Aussagewert zu haben. Die von den Medizinern angegebenen Fristen schwanken -zumeist unter mehrfacher Messungzwischen 3 und 48 Stunden. Die Deutsche Geselschaft für Chirurgie verlangt zwei im Abstand von 12 Stunden abgenommene, jeweils einstïndige Electro-Encephalogramme mit ununtebrochener Null-Linie ${ }^{15}$.

Die anfängliche wohl nicht vermutete Unzuverlässigkeit des EEG hat zur Aufstellung einer Reihe von Kriterien geführt, die neben der in Sicherheitsabständen mehrfach gemessenen NullLinie des EEG vorliegen müssen, um den Nachweis des (primären) Gehirntodes zu erbringen. Nach der im wesentlichen übereinstimmenden Ansicht medizinischer Gesellschafiten und namhafter medizinischer Autoren müssen über einen längeren Zeitraum (von mindestens 12 Stunden) hinweg festgestellt werden : andauernde tiefe Bewußtlosigkeit, völliger Ausfall jeglicher Spontanatmung sowie weite und lichtstarre Pupillen. Häufig werden außerdem völliger Ausfall sonstiger Eigen- und Fremdreflexe, nicht selten auch rascher Blutdruckabfall and Erlöschen der Temperaturregulation

14 Vgl. zum folgenden: Stratenwerth (wie Anm. 4), S. 545, mit Nachw.

15 Vgl. dazu und zum folgenden: Die in Anm. 5 zitierte EntschlieBung der Deutschen Gesellschaft für Chirurgie. 
gefordert. Dieser summarische Hinweis mag genügen, um das erwähnte ärztliche Gesamturteil bei der Hirntodfeststelleung anschaulich zu machen.

b) Die Langwierigkeit dieses Verfahrens hat begreiflicherweise $\mathrm{zu}$ medizinischen Bemühungen um andere und schnellere diagnostische Hilfsmethoden für den Nachweis des Gehirntodes geführt. Dabei geht es - unter anderem- um folgende zwei Ver. fahren :

Einerseits versucht man, nach Einspritzung eines Kontrastmittels in die zum Gehirn führenden Schlagadern röntgenologisch das Aufhören des Blutkreislaufs im Gehirn festzustellen (= beidseitige Carotis- und Vertebralis-Angiographie); nach einem die Uberlebensfrist des Gehirns übersteigenden und sicherheitshalber auf 30 Minuten bemessenen Kreislaufstillstand wäre das Gehirn mit Sicherheit tot. Aber zunächst ist diese Methode nicht allgemein zum Nachweis des Gehirntodes anwendbar, sondern nur dann, wenn der Hirntod auf ganz bestimmten Ursachen beruht, nämlich auf einer direkten Gehirnschädigung durch Gewalteinwirkung oder durch intrakranielle Drucksteigerung. Vor allem aber bestehen bei diesem anfwendigen Verfaihren noch technische Fehlerquellen, die den Wert dieser Methode mindern. ${ }^{16}$-Anderseits versucht man, die Sauerstoffdifferenz im Blut der zum Gehirn hinführenden und der von ihm wegführenden Blutgefäße zu messen, um so den zerebralen Sauerstoffverbrauch -beziehungsweise die Unterschreitung oder den gänzlichen Ausfall des Erhaltungsstoffwechselsfestzustellen; auch hier müßte theoretisch nach einer Sicherheitsfrist der Gehirntod nachweisbar sein. Aber dieses komplizierte Verfahren zur Blutgasanalyse hat bisher noch nicht zu gesicherten Ergebnissen geführt. ${ }^{16}$ - Bei beiden Methoden sind also zumindest gleichfalls zusätzliche klinisch-neurologische Untersuchungen auf Hirntodsymptome nötig.

Vor allem aber stoßen diese beiden Unteruchungensmethoden auf erhebliche rechtliche Bedenken. ${ }^{17}$ Sie machen nämlich größere körperliche Eingriffe beim Patienten nötig, die zudem mit Risiken verbunden sind ${ }^{18}$. Im Augenblick ihrer Vornahme steht aber der Tod des Patienten gerade noch nicht fest, mögen auch einzelne

\footnotetext{
${ }^{16}$ Vgl. dazu : Bay in Bay-Römer, Medizinische und rechtliche Aspekte von Organverpflanzungen, 1970, $\mathbf{S}$. 6 ff.

17 Vgl. zum folgenden: Geilen, JZ 1971, S. 42.

18 Vgl. Bay wie Anm. 16.
} 
der genannten Kriterien schon für das Vorliegen eines Hirntodes sprechen. Jeder sachkundige und verantwortungsbewu $\beta$ te Arzt mu $\beta$ also in Rechnung stellen, da $\beta$ der. Patient zur Zeit dieser Eingriffe noch lebt, mag er dies in Kauf nehmen oder es außer acht lassen (bedingter Vorsatz oder Fahrlässigkeit). Daraus ergeben sich dann rechtliche Komplikationen : Die deutsche Rechtsprechung wentet nämlich jeden ärztlichen Eingriff am Körper eines (lebenden) Menschen als Körperverletzung (\$§ $223 \mathrm{ff}, 230$ StGB), die folglich eines Rechtfertigungsgrundes bedarf. Dem stimmt die detutsche Strafrechtslehre einhellig für diejenigen Fälle zu, in denen es sich nicht um Heileingriffe im Interesse des Patienten selbst, sondern um Eingriffe in fremden Interesse handelt. Letzteres ist aber hier der Fall, denn es geht ja um Eingriffe, mittels deren beschleunigt festgestellt werden soll, ob der Tod schon eingetreten ist, um dann Organe aus dem Spender zur Transplantation auf einen Empfänger entnehmen zu können. Eine Rechtfertigung dieser -der Hirntodfeststellung dienenden- körperverletzenden Eingriffe am noch lebenden Patienten ist aber nach deutschem Recht so gut wie ausgeschlossen: Der Rechtfentigungsgrund des sogenannten «übergesetzlichen Notstands» scheidet aus. Denn nach herrschender Ansicht erlaubt der beim übergesetzlichen Notstand anzustellende Wertvergleich es schon im Hinblick auf die Menschenwïde nicht, den Körper eines anderen unter Nichtachtung seiner Freiheitsrechte als bloßes Mittel zur Erreichung eines -wenn auch erwünschten-Zweckes zu verwenden ${ }^{19}$. Das gilt nach weit über* wiegender Ansicht schon für relativ harmlose Eingriffe wie eine Blutentnahme zum Zwecke der Bluttransfusion; es mu $\beta$ aber sicher und erst recht für fremdnützige risikoreiche Eingriffe an Schwerkranken und Sterbenden gelten. -Der einzige theoretisch denkbare Recht fertigungsgrund der «Einwilligung» des Patienten scheidet praktisch aus. Denn in dieser Situation kann von der rechtswirksamen Erteilung einer Einwilligung des (bewußtlosen!) Patienten naturgemäß nicht mehr die Rede sein. Und da $\beta$ jemand im vorhinein wirksam in etwaige spätere Eingriffe dieser Art einwilligen wïde, ist gewi $\beta$ reine Theorie.

Es muß daher Verwunderung und Befremden erregen, mit welcher Unbefangenheit solche Methoden zur Feststellung des Gehirntodes praktiziert und teilweise auch von medizinischen Ge sellschaften anerkannt werden, ohne da $\beta$ eine tragfähige rechtliche Grundlage dafür besteht.

19 Eingehende Nachweise bei Lüttger in JR 1971, S. 309 ff. 
2. Doch kehren wir noch einmal zurück zu der erstgenanten Methode, derzufolge der Gehirntod durch die geschilderten (essentiellen) klinisch-neurologischen Symptome in Verbindung mit der EEG-Kontrolle nachgewiesen wird; denn dort stellt sich noch ein weiteres Beweisproblem. Da nämlich diese Kriterien nur in ihrer Gesamtineit den Gehirntod beweisen, haben sich einige medizinische Regeln gebildet, die auch in juristischer Sicht von Interesse sind; sie besagen ${ }^{20}$ :

Grundsätzlich ist davon auszugehen, da $\beta$ die Funktionen von Kreislauf und Atmung - unabhängig davon, ob küinstlich oder spontan- solange das Bestehen des Lebens beweisen, bis der Gegenbeweis --der Nachweis des Erlöschens der Hirnfunktion- erbracht ist. Und weiter : Die Null-Linie im EEG hat nur Beweiskraft, solange ihr nicht andere intakte Körperfunktionen entgegenstehen, wie Spontanatmung, normale Herztätigkeit, erhaltene Reflexe oder ein Weiterbestehen des (wenn auch geistig gestörten) Bewußtseins. Im übrigen widerlegt jede —noch so geringe- Wellenaktivität im EEG den Organtod des Gehirns ohne weiteres. Und es gilt ganz allgemein die ärztliche Sorgfaltsregel, daß beim leisesten Zweifel an der Eindeutigkeit des Befundes "in dubio pro patiente" ein Weiterbestehen des Lebens anzunehmen ist. - Schließlich wird von medizinischer Seite noch eine zweifache Vorsichtsmaßnahme personeller Art gefordert: Zur Feststellung des Gehirntodes sollen neben den die übrigen Symptome prüfenden Ärten noch Spezialisten zugezogen werden, so ein Neurologe oder Neurochirurg zur Beurteilung der Hirnfunktionen und ein EEG-Fachmann zur Begutachtung des Hirnstrombildes. Ferner sollen die den zerebralen Tod feststellenden Ärzte vom Transplantationsteam unabhängig sein, um seine getrennte ärztliche Verantwortlichkeit für Organspender und Organempfänger zu gewährleisten.

Die letztgenannten personelen Vorsichtsmaß̣nahmen können zwar de lege lata rechtlich nicht erzwungen werden, weil unser Recht kein bestimmtes Verfahren für die ärtliche Todesfeststellung vorschreibt ${ }^{21}$. Die übrigen - so treffend in der Formel «in dubio pro patiente» gipfelnden- medizinischen Sorgfaltsregeln thaben jedoch zugleich einen triftigen juristischen Grund. Solange nämlich nicht eimwandfrei feststeht, da $\beta$ der Patient, dem ein

${ }^{20}$ Eingehende Nachweise zum folgenden bei : Lüttger in JR 1971, S. 309 ff.

21 Vg1. dazu näher: Geilen in JZ 1971, S. 42 ff. 
Transplantat entnommen werden soll, hirntot ist, besteht für den Arzt ein schwerwiegendes strafrechtliches Risiko :

Stellt sich beispielsweise heraus, da $\beta$ der Spender zur Zeit des Eingriffs noch lebte und da $\beta$ er erst durch den Eingriff getötet worden ist, so kommt eine strafrechtliche Haftung des Arztes unter zwei Gesichtspunkten in Betracht: Hat der Arzt es bei seinem Eingriff für möglich gehalten, da $\beta$ der Spender noch lebte, und hat er dies zustimmend in Kauf genommen, so ist er wegen eines mit dolus eventualis begangenen vollendeten Totschiags (oder gar Mordes) strafbar. Hat der Arzt jedoch einfach songlos darauf vertraut, da $\beta$ der Spender schon tot sei, so ist er wegen fahrlässiger Tötung strafbar. -Aber selbst dann, wenn sich herausstellt, $\mathrm{da} \beta$ der Spender bei dem Eingriff bereits tot war und da $\beta$ die Organentnahme mithin aus einer Leiche erfolgt ist, so ist das strafrechtliche Risiko des Arztes gleichwohl nicht ausgeräumt: Hat der Arzt es nämlich für möglich gehalten und in Kauf genommen, daß der Spender noch lebte, so ist er wegen versuchten Totschlags am untauglichen Objekt strafbar. Hat er hingegen in diesem Falle sorglos darauf vertraut, da $\beta$ der Spender wohl schon tot sein werde, so entfällt freilich eine strafrechtliche Haftung, weil der Versuch eines. Fahrlässigkeitsdelikts nicht strafbar ist. Jedenfalls aber erweisen sich die geschilderten medizinischen Sorgfaltsregeln auch in juristischer Sicht als wohlbegründet.

Ist jedoch der Gehirntod einwandfrei diagnostiziert, so klären sich manche Rechtsfragen: Ein rechtlicher Zwang, Wiederbelebungsversuche anzustellen, kommt nicht in Betracht, und cin schon laufender Reanimationsproze $\beta$ darf abgebrochen werden; denn einem «Toten» gegenüber entfällt jede ärztliohe Hilfeleistungsflicht. Und die Entnahme von Transplantaten aus dem Körper eines Hirntoten stellt weder eine Tötung noch eine Körperverletzung dar. Der Arzt hat nur noch die zum Schutze der Leichen bestehenden Vorschriften zu beachten. ${ }^{23}$

c.

Bedenkt man die vielfältigen Probleme und Schwierigkeiten, die mit der Anerkennung und Feststellung des Hirntodes verbunden sind, so kann es nicht verwundern, da $\beta$ der Ruf nach dem

\footnotetext{
${ }^{22} \mathrm{Im}$ deutschen Strafrecht ist seit Generationen unbestritten nich nur der Versuch mit untauglichen Mitteln, sondern auch der Versuch am untaug. lichen Objekt strafbar.

${ }^{23}$ Vgl. zu dieser Problematik näher: Dreher, Kommentar zum Strafgesetzbuch, 32. Auflage 1970, Anmerkung 2 B zu $\S 168$ StGB.
} 
Gesetzgeber laut geworden ist. Manche Autoren haben eine gesetzliche Festlegung des Todesbegriffs beziehungsweise der Todeskriterien gefordert; zahlreiche andere Autoren haben diesem Verlangen entschieden widersprochen. Auch ich habe Bedenken gegen diese Forderung, und zwar aus folgenden Gründen :

Der "offene Rechtsbegriff» des Todes wird -wie ich dargelegt habe- durch die medizinische Wissenschaft ausgefüllt. Er ist daher dem inhaltlichen Wandel durch neue medizinische Erkenntnisse unterworfen. Das hat sich gerade jetzt bei der Ersetzung der klassischen Todesdefinition des «Herz- und Atmungstodes» durch den neuen Begriff des "Gehirntodes» eindrucksvoll gezeigt. Für eine Anerkennung des Hirntodes als Tod im Rechtssinne bedarf es also keines Gesetzes.

Geradezu verhängnisvoll aber wäre es, wenn der Gesetzgeber die medizinischen Kriterien für den Nachweis des Gehirntodes festlegen wollte. Die medizinische Wissenschaft ringt gerade jetzt und wohl noch lange Zeit um seine Verbesserung dieser Kriterien und ihres Beweises; auf lange Sicht wird es bei einer sich ständig wandelnden Zwischenbilanz dieser Forschungen bleiben. Daher wäre es offensichtlich verfehlt, den derzeitigen Stand dieser medizinischen Zwischenbilanz in Gesetzesform zu versteinern. Ein Gesetz, das die Symptome des Gehirntodes und die Anforderungen an ihren Nachweis festlegen wollte, wäre schon im Augenblick seiner Verkündung überholt. Aber den Gleichschritt des Rechts mit dem Fortschritt der Medizin hätte es torpediert; und sehr wahrscheinlich würde es sogar den Fortschritt der Medizin behindern. Der vermeintliche Gewinn an Rechtssicherheit wäre mit der Wissenschaftsfeindlichkeit einer solchen Gesetzgebung viel zu teuer bezahlt. Auf absehbare Zeit sollte es Sache der ärztlichen Fachorganisationen bleiben, durch laufende Anpassung ihrer Richtlinien über die Kriterien des Hirntodes an den gesicherten Forschungsstand die lex artis in diesem Bereich kundzutun.

Aber auch ohne Festlegung von Todesbegriff und Todeskriterien gibt es zahlreiche Rechtsfragen, die ein -in der Bundesre publik Deutschland dringend nötiges- Transplantationsgesetz regeln könnte $e^{24}$. Das beginnt bei Verfahrensfragen, wie etwa der Einschaltung unabhängiger Ärzteteams bei der Feststellung des Hirn-

${ }^{24} \mathrm{Vgl}$. zum folgenden: Bockelmann (wie Anm. 13), S. 118; Heinitz (wie Anm. 4), S. 30. 
todes. Es setzt sich fort mit materiellen Fragen; etwa bei der Überlegung, ob eine Rechtsgrundlage für die geschilderten, de lege lata rechtlich höchst bedenklichen Untersuchungsmethoden geschaffen werden kann. Und es geht hin bis zu der nachgerade überfälligen Regelung der in der Bundesrepublik Deutschland heftig umstrittenen Voraussetzungen fïr die Entnahme von Transplantaten aus einer Leiche. ${ }^{25}$ Wir sollten uns mit dem Erreichbaren und Nötigen begnügen, nicht aber nach den Sternen greifen.

25 Vgl. dazu Anm. 23. 